\title{
Fate of many-body localization under periodic driving
}

\author{
Achilleas Lazarides ${ }^{1}$, Arnab Das ${ }^{2}$ and Roderich Moessner ${ }^{1}$ \\ 1 Max-Planck-Institut für Physik komplexer Systeme, 01187 Dresden, Germany and \\ 2 Theoretical Physics Department, Indian Association for the Cultivation of Science, Kolkata 700032, India
}

\begin{abstract}
We study many-body localised quantum systems subject to periodic driving. We find that the presence of a mobility edge anywhere in the spectrum is enough to lead to delocalisation for any driving strength and frequency. By contrast, for a fully localised many-body system, a delocalisation transition occurs at a finite driving frequency. We present numerical studies on a system of interacting one-dimensional bosons and the quantum random energy model, as well as simple physical pictures accounting for those results.
\end{abstract}

Introduction-The study of disorder and localisation has a long and productive history, beginning with the seminal work of Anderson 11. More recently, the effects of disorder on interacting systems have been considered under the heading of many-body localisation (MBL) 1, 2, in part motivated by fundamental questions relating to thermalisation in closed quantum systems.

At the same time, significant theoretical effort has been devoted to understanding thermalisation in periodically driven systems. There has been work recently on the long-time behaviour of both integrable [4, 5] and nonintegrable [2, 3, 8] (with Ref. [8] also studying locally driven MBL systems). For clean systems or MBL systems in their delocalised phase, it has been found that driving leads to a state equivalent to a fully-mixed state, satisfying a special case of the Eigenstate Thermalisation Hypothesis (ETH, see [2, 3, 8, 12]). Local periodic driving of MBL systems in their localised phase, on the other hand, has been argued not to have any global effects [8].

In this work, we study the effects of global periodic

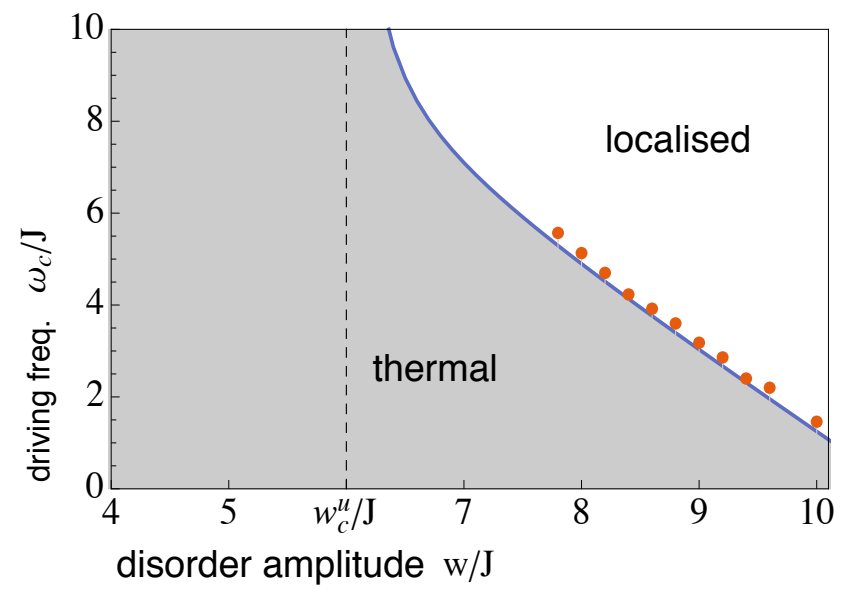

Figure 1: Plot of driving frequency $\omega_{c}$ below which the system delocalises as a function of disorder amplitude $w$. The shaded areas correspond to delocalisation. The red dots are obtained from finite-size studies of the level statistics of the system. The disorder amplitude $w_{c}$ is the value below which the undriven system is delocalised in the absence of driving. The blue line is a guide to the eye.

\begin{tabular}{|c|c|c|}
\hline Mobility edge & low frequency & high frequency \\
\hline \hline present & delocalised & delocalised \\
\hline absent & delocalised & localised \\
\hline
\end{tabular}

Table I: Effect of driving frequency in the presence and absence of a mobility edge

driving, and find that there exists a regime where MBL survives. We identify two mechanisms by which periodic driving might destroy MBL, depending on the existence or otherwise of a mobility edge. The first, rather robust, mechanism is the mixing of undriven eigenstates from everywhere in the spectrum by the driving; if there is a mobility edge, this results in delocalisation of all states of the effective Hamiltonian. The second mechanism is more subtle and involves strong mixing of states [2] which cause a delocalisation transition at finite frequency. Our findings are summarised in Table 1 .

In what follows, we begin by studying the case of no mobility edge. We introduce and numerically solve a system described by a local non-integrable Hamiltonian. After establishing the existence of the aforementioned critical frequency using level statistics, we demonstrate that ETH is (is not) satisfied below (above) this frequency, and present a physical picture explaining this phenomenon. We then move to the case where a mobility edge exists. As a case study, we use the Quantum Random Energy Model (QREM) which has recently been shown to display a mobility edge. A direct numerical solution confirms that driving delocalises the entire spectrum, consistent with an intuitive argument we sketch. Finally, we point out open questions.

We shall concentrate throughout on systems described by Hamiltonians of the form

$$
H(t)=H_{0}+H_{D}(t)
$$

so that their time evolution is described by an effective Hamiltonian $H_{e f f}(\epsilon)$ for each instant $\epsilon$ during the period $T$, defined by

$$
\exp \left(-i H_{\text {eff }}(\epsilon) T\right)=\mathcal{T} \exp \left(-i \int_{\epsilon}^{\epsilon+T} d t H(t)\right) .
$$




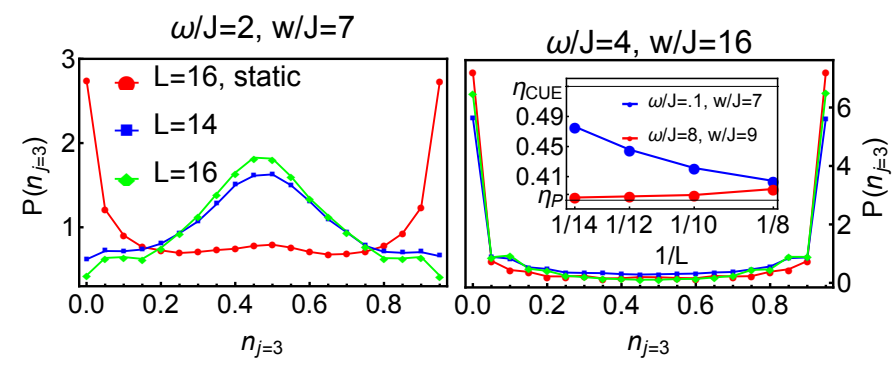

Figure 2: Main plot: Probability distribution of the eigenstate expectation values (EEVs) of the density at site $j=3$. Left (right): Driving with a low (high) frequency (see Fig. 1) results in the probability distributions which does (does not) develop a central peak upon increasing system size, signalling delocalisation 2]. Data is disorder averaged over $10^{4}(100)$ realisations for $L=14(L=16)$. Inset: Level statistics parameter versus inverse system size in the localised (bottom, red) delocalised (top, blue) phases. The parameter $\eta=\int d s s P(s)$ with $P(s)$ the probability distribution of the level statistics [1] 3], taking the value $\eta_{P / C U E}$ in the localised/delocalised regime. Data averaged over 1000 disorder realisations for $L=8,10,12,100$ realisations for $L=14$.

Without loss of generality we set $\epsilon=0$ (see Ref. [4]). The eigenvalues of $H_{\text {eff }}(\epsilon)$, called the quasienergies, are independent of $\epsilon$ and effectively play the role of energy eigenvalues.

We now define what we mean by localised and delocalised phases. In a localised phase, the (quasi-)energy level statistics do not display level repulsion, and the expectation values of operators in the eigenstates of the (effective) Hamiltonian fluctuate wildly from eigenstate to eigenstate. In a delocalised phase, the opposite is true: the levels repel each other, and the expectation values of physical, local operators in nearby energy or quasienergy states are similar. Other definitions are possible and, in general, equivalent (see eg Ref. [13]). The connection between the eigenstates of $H_{\text {eff }}$ and the applicability of ETH was elucidated in Ref. [2]. The framework developed there turns out to be natural for discussing the case of a system which, in the absence of driving, is in the MBL phase.

No mobility edge: Local Model-Let us introduce a model of interacting hard-core bosons described by a driven, local Hamiltonian (Eq. 1) with

$$
H_{0}=H_{h o p}+\sum_{r=1}^{2} V_{r} \sum_{i=1}^{L-1} n_{i} n_{i+r}+\sum_{i=1}^{L} U_{i} n_{i}
$$

where $H_{\text {hop }}=\left(-\frac{1}{2} J \sum_{i=1}^{L-1}\left(b_{i}^{\dagger} b_{i+1}+b_{i+1}^{\dagger} b_{i}+h c\right)\right)$ is a hopping operator, the $b$ are hard-core bosonic operators, $U_{i}$ an on-site random potential uniformly distributed between $-w$ and $+w$ and $H_{D}(t)$ a time-periodic hopping term

$$
H_{D}(t)=\delta \tilde{\delta}(t) H_{h o p}
$$

with $\delta$ a dimensionless constant, $\tilde{\delta}(t)=-1(+1)$ in the first (second) half of each period $T=2 \pi / \omega$. Via Jordan-Wigner transformations this model is related to a fermionic interacting system as well as to a spin- $1 / 2$ chain. Throughout this work we will concentrate on the specific case $V_{1} / J=V_{2} / J=1$, although our qualitative conclusions are not sensitive to this.

To locate the transition in the undriven model we use the standard technique [1] involving finite-size scaling of the level statistics (see inset of Fig. 6 and Supplemental Material). At half-filling there thus appears to be a transition at a disorder amplitude $w_{c}^{u} / J(\approx 6$ for our interaction parameters $V_{1} / J=V_{2} / J=1$ ) [30].

We now drive this system $\delta \neq 0$. The level statistics of the quasienergies of $H_{\text {eff }}$ (Eq. 2), show level repulsion in the clean limit [3] but are found to cross freely (indicating localisation) in the MBL regime if driven locally, as reported in Ref. 8]. Here, we show that globally periodically driving the system in the MBL regime delocalises the system if the driving frequency is below a (system size independent) critical value. We argue that this is a consequence of the structure of the effective Hamiltonian for an MBL system [14 18.

As established above, the undriven system is in the delocalised phase for disorder amplitude $w<w_{c}^{u}$; driving at this disorder is qualitatively similar to driving any nonintegrable system 2, a case that has been studied in Ref. [2]. We have indeed confirmed quasienergy level repulsion for $w<w_{c}^{u}$.

To study the MBL regime, $w>w_{c}^{u}$, we switch on periodic driving (Eq. 4) with amplitude $\delta / J=0.1$ (our results do not change qualitatively for different $\delta$ provided the system is large enough that the local level spacing is less than $\delta$ ). We directly calculate $H_{\text {eff }}$ and its level statistics. As our central result we find that for each disorder amplitude there exists a driving frequency $\omega_{c}(w)$ above which the system remains in the localised phase under driving (see Supplemental Material), while for $\omega<\omega_{c}(w)$ the system delocalises. This frequency is plotted in Fig. 1 as a function of disorder amplitude $w$, while examples of the level statistics results are shown in the inset of Fig. 6. We expect $\omega_{c}(w)$ to diverge as $w$ approaches $w_{c}^{u}$ from above.

Having established a transition via the level statistics, we now show in addition that the phases above (below) $\omega_{c}$ do (do not) satisfy the form of ETH discussed in Ref. [2, further reinforcing our interpretation of $\omega_{c}$ as a "delocalisation frequency". We consider a localised undriven system and provide in Fig. 6 direct evidence for the fullymixed nature of the eigenstates of $H_{\text {eff }}$ for slow - but not for fast - driving. The quantity under consideration is the probability distribution for the eigenstate expectation values (EEVs) [2] of the density operator. Driving faster than the delocalisation frequency (right panel) yields little change in the probability distribution. By contrast, driving slowly (left panel), a central peak is seen to de- 


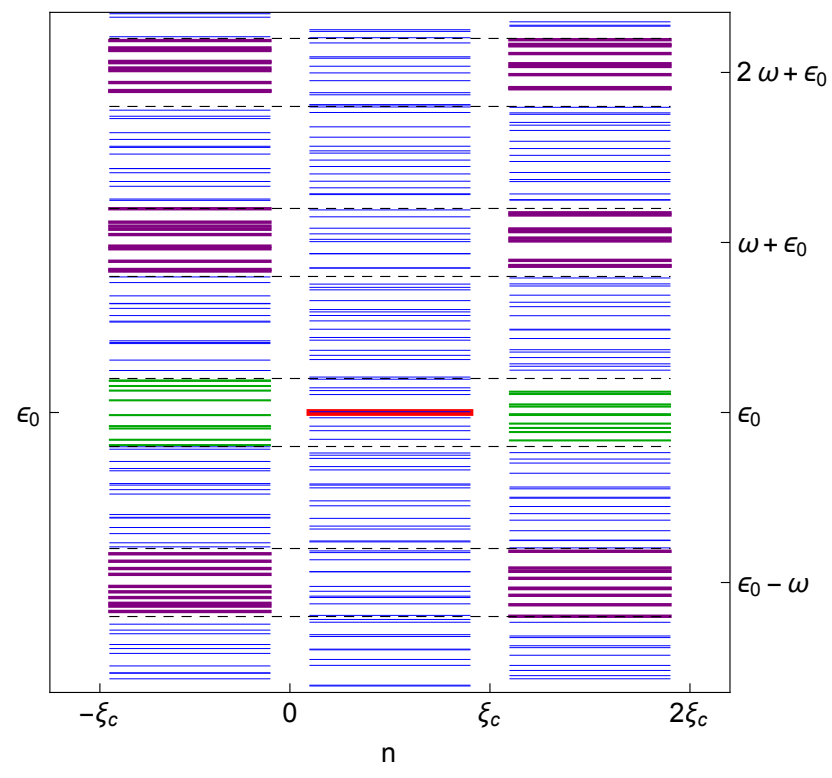

Figure 3: Schematic illustration of sub systems and their energy levels in an MBL system. The horizontal axis indexes the conserved quantity (increasing $n$ corresponds to increasing spatial index $i$ ); the vertical indexes energy. $\xi_{c}$ is some localisation length, setting the typical spatial size of the subsystems. A periodic coupling of the subsystems with frequency $\omega$ couples the red state in the middle block to both the purple and the green levels in the two neighbouring blocks, while a time-independent coupling would only couple it to the green ones. The width of the purple and green strips is set by the amplitude of the driving. Critically, the limit of $\omega$ greater than the typical subsystem bandwidth is indistinguishable from a time-independent driving. By contrast, the limit of $\omega \rightarrow 0$ collapses the local spectra, wiping out the effect of disorder.

velop with increasing system size, corresponding to the EEVs all being equal and given by $n_{j=3}=0.5$. This is the fully-mixed result for our system at half-filling, corresponding to delocalisation [2].

In passing, let us remark that, since the EEVs of the instantaneous Hamiltonian show the same behaviour as in Fig. 66 (data not shown), our localised phase is not unlike the localisation in energy space discussed in Ref. [19], even though the underlying physics is quite different.

Physical picture-We now relate our numerical findings to a physical picture valid for weak driving. In the MBL phase and in the absence of driving, the system is effectively integrable in that there exist extensively many local integrals of motion [14-18. The system may thus be thought of as a set of local subsystems, of finite spatial extend, therefore of finite energetic bandwidth as schematically shown in Fig. 3. As a result, if the driving frequency is larger than the typical local subsystem bandwidth, the system cannot absorb energy from the driving and does not react. Therefore, driving with a frequency much higher than the typical local bandwidth cannot destroy $M B L$. In contrast low-frequency driving may be understood by viewing our driving protocol as a series of quenches: as MBL systems eventually reach a steady-state after an instantaneous quench [21, 22, periodic driving with the protocol we use can be thought of as a series of non-adiabatic perturbations. It is quite natural then to expect this to cause the system to spread in energy space, delocalising it.

Let us elaborate this pair of arguments, beginning with high-frequency driving.

High-frequency driving-The most general form of $H_{M B L}$ consistent with known phenomenology such as vanishing of the conductivity at all energies is

$$
H_{M B L}^{G}=\sum_{n} \mathcal{H}_{n}^{(\ell)}+\sum_{m<n} \mathcal{H}_{n}^{(\ell)} V_{m, n}^{(\ell)} \mathcal{H}_{m}^{(\ell)} \ldots
$$

with the $\mathcal{H}_{n}^{(\ell)}$ Hamiltonians for local subsystems (with local spatial support) and $n$ a spatial index indicating the site about which the subsystem is centred [14-18. Due to its locality, each $\mathcal{H}_{n}^{(\ell)}$ has a local spectrum of some typical, finite width set by the disorder amplitude and other system details and independent of the other blocks (see Fig. 3, where the spectra for three $\mathcal{H}_{n}^{(\ell)}$ are sketched schematically).

Driving $H_{M B L}^{G}$ with a sum of local terms such as in Eq. 11 couples each $\mathcal{H}_{n}^{(\ell)}$ to its neighbours 31] via terms allowing energy and matter transfer. Consider a single energy level for $n=0$ (middle block, Fig. 3), indicated by the red line in the middle block. A timeindependent coupling between the blocks couples it to the green blocks on each side, while a periodic coupling with frequency $\omega$ couples it to both the green and purple blocks by virtue of folding the energy spectrum into the $\omega$-periodic quasienergies. Crucially, for $\omega$ larger than the typical width of the blocks, folding the local spectra has no effect [20] and a weak coupling does not delocalise the system, as it acts similarly to a time-independent perturbation [32. In other words, the system can react to the driving by absorbing energy quanta $\omega$ only if there exist levels separated by this energy. In the presence of MBL the typical local bandwidth sets the maximum driving frequency to which the system can react. 33.

Low frequency- In the limit of low-frequency driving disorder is effectively suppressed and the delocalised phase is always reached.

This phenomenon is best understood in the time domain as follows. Consider time evolving with Hamiltonian $H_{1(2)}$ for the first (second) half of the period. This series of nonadiabatic changes to the system generically results in a broadening of the energy distribution, provided that the half-period $T / 2$ is longer than the characteristic relaxation time [21, 22]. Typically, this eventually leads to a fully-mixed state occupying the entire Hilbert space equiprobably.

There are two central ingredients to this argument. The first is that the relaxation time does not diverge with 

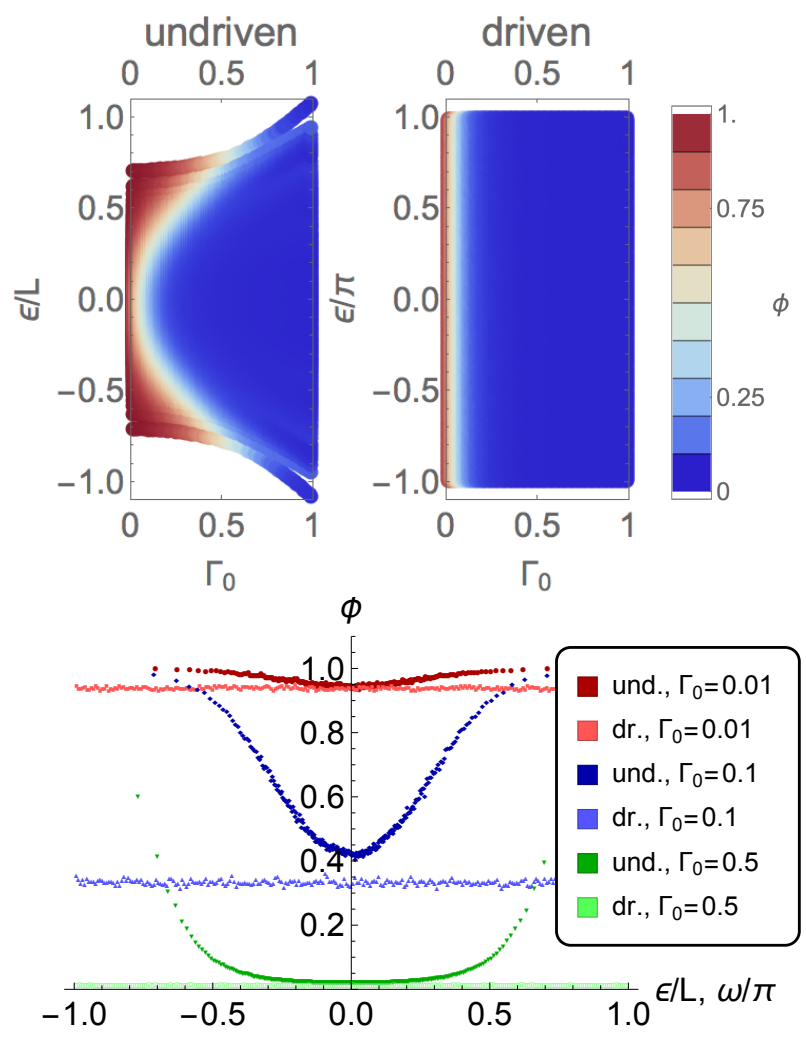

Figure 4: Driving the QREM. The top left figure shows the participation ratio $\phi$ for the eigenstates of the undriven model, showing a mobile region (blue) surrounded by a localised region (red). Driving with frequency $\omega / J=0.1$ and amplitude $\delta / J=0.2$ (top right) causes all states at a given $\Gamma_{0}$ to become as delocalised as the least localised state at that $\Gamma_{0}$ in the undriven model. This is also shown in the bottom panel which shows $\phi$ for $\Gamma_{0}=0.01,0.1,0.5$ (red, blue and green line, from top to bottom) in the absence (presence) of driving with darker (lighter) colour. The driven points always lie below the undriven points for the corresponding $\Gamma_{0}$. This is due to the strong mixing of all undriven eigenstates by the driving. All data in this figure is for 8 spins and averaged over 1000 disorder realisations.

system size so that the half-period $T / 2$ can be longer. The existence of a dephasing timescale independent of system size [21, 22] ensures that this is the case. The second is that repeatedly dephasing in the two different eigenstate bases does lead to energy delocalisation. Since $H_{1,2}$ are both MBL Hamiltonians, the eigenstates of one are in general localised in terms of the eigenstates of the other. Nevertheless, repeated cycles of dephasing to alternating bases do indeed eventually lead to a fully-mixed state, as is shown in the Supplemental Material.

A mobility edge: The QREM as a case study-We now turn to the case in which a mobility edge is present in the undriven spectrum. Our central result is based on the observation 2 2 that a periodic perturbation acting on a system couples each undriven state to states spread uniformly throughout the spectrum of $H_{0}$. As a result, if part of the spectrum corresponds to delocalised eigenstates then all eigenstates of $H_{\text {eff }}$ will necessarily be delocalised. We numerically confirm this by studying the Quantum Random Energy Model (QREM), recently studied in Ref. 23. where it was shown to have a mobility edge. This model is described in Ref. [23]: it is defined for $N$ Ising spins with the Hamiltonian $H=E\left(\left\{\sigma_{j}^{z}\right\}\right)-\Gamma \sum_{j} \sigma_{j}^{x}$, where $E$ is a random operator diagonal in the $\sigma^{z}$ basis (that is, it assigns a random energy to each spin configuration) and $\Gamma$ a transverse field. Extensivity of the many-body spectrum is satisfied if the random energies are drawn from a distribution $P(E)=\frac{1}{\sqrt{\pi N}} \exp \left(-E^{2} / N\right)$.

The diagnostic of localisation we use is the participation ratio (PR), defined for the state $|\psi\rangle$ as $\phi=$ $\sum_{n}|\langle n \mid \psi\rangle|^{4}$ with $n$ enumerating Fock states. $\phi$ approaches unity for a state localised on a single Fock state and $2^{-N}$ for one fully delocalised in Fock space. The leftmost panel shows in Fig. 4 shows the average $\phi$ versus energy (scaled with system size) of the 256 eigenstates of an undriven $N=8$ system averaged over 1000 disorder realisations, demonstrating the existence of a mobility edge.

Next, we drive the system by modulating $\Gamma(t)=$ $\Gamma_{0}(1+\delta \tilde{\delta}(t)), \tilde{\delta}(t)=+1(-1)$ for the first (second) half of the period with an amplitude $\delta=0.2$ and frequency $\omega=2 \pi / T=0.1$. The PR of the eigenstates of $H_{\text {eff }}$ are shown in the second panel of Fig. 4. As expected, periodic driving causes delocalisation of the entire spectrum so long as part of the undriven spectrum at the same $\Gamma_{0}$ is delocalised.

Outlook-We have shown that many-body systems can remain many-body localised, with Poissonian level statistics, when they are subjected to slow driving. On the other hand, for fast driving or in the presence of a mobility edge, delocalisation will occur, with driving inducing level repulsion.

This "classification" of the behaviour of MBL systems under driving immediately raises further questions. What are the timescales involved in reaching the longtime state we have discussed, how do they depend on the driving amplitude and frequency, and how do they differ between the localised and the delocalised limit? What is the precise difference between local and global driving as far as both the long-time state and the approach to it are concerned? More broadly, we have concentrated on systems with a bounded local spectrum. What happens if it is unbounded, as in the cases of a continuum system or of a lattice boson system? What if we bring the system in contact with a heat bath?

We believe that the dual out-of-equilibrium situation driving and MBL - is only beginning to be explored and will prove to be fertile ground for future research.

Acknowledgements-We thank J. Bardarson, M. Haque, V. Khemani, J. Kjall, V. Oganesyan, S. Sondhi, O. Tiele- 
man and particularly T. Scheler for discussions as well as D. Abanin, A. Chandran and L. D'Alessio for exchanges in the course of this project.

Note added: After the completion of this work, two related works [25, 26] have appeared. Each of these takes a somewhat different perspective but they all establish phenomenologies essentially consistent with the one we report.

[1] P. W. Anderson, Phys. Rev. 109, 1492 (1958).

[2] D. M. Basko, I. L. Aleiner, and B. L. Altshuler, Ann. Phys. 321, 1126 (2006).

[1] V. Oganesyan and D. Huse, Phys. Rev. B 75, 155111 (2007)

[4] A. Lazarides, A. Das, and R. Moessner, Phys. Rev. Lett. 112, 150401 (2014)

[5] A. Russomanno, A. Silva, and G. E. Santoro, Phys. Rev. Lett. 109, 257201 (2012).

[3] L. D'Alessio and M. Rigol, (2014), arXiv:1402.5141.

[2] A. Lazarides, A. Das, and R. Moessner, Phys. Rev. E 90, 012110 (2014).

[8] P. Ponte, A. Chandran, Z. Papić, and D. A. Abanin, (2014), arXiv:1403.6480.

[9] J. Deutsch, Phys. Rev. A 43, 2046 (1991)

[10] M. Srednicki, Phys. Rev. E 50, 888 (1994).

[11] M. Rigol, V. Dunjko, and M. Olshanii, Nature 452, 854 (2008)

[12] W. Beugeling, R. Moessner, and M. Haque, Phys. Rev. E 89, 042112 (2014)

[13] J. A. Kjäll, J. H. Bardarson, and F. Pollmann, Phys. Rev. Lett. 113, 107204 (2014).

[14] D. A. Huse, R. Nandkishore, and V. Oganesyan, (2014), arXiv:1408.4297.

[15] A. Chandran, I. H. Kim, G. Vidal, and D. A. Abanin, (2014), arXiv:1407.8480.

[16] M. Serbyn, Z. Papić, and D. A. Abanin, Phys. Rev. Lett. 111, 127201 (2013)

[17] V. Ros, M. Mueller, and A. Scardicchio, (2014), arXiv:1406.2175.

[18] J. Z. Imbrie, (2014), arXiv:1403.7837.

[19] L. D'Alessio and A. Polkovnikov, Ann. Phys. 333, 19 (2013).

[20] A. Eckardt, T. Jinasundera, C. Weiss, and M. Holthaus, Phys. Rev. Lett. 95, 200401 (2005).

[21] R. Vasseur, S. A. Parameswaran, and J. E. Moore, (2014), arXiv:1407.4476.

[22] M. Serbyn, Z. Papic, and D. A. Abanin, (2014), arXiv:1408.4105.

[23] C. R. Laumann, A. Pal, and A. Scardicchio, (2014), arXiv:1404.2276.

[24] A. Roy and A. Das, Phys. Rev. B 91, 121106(R) (2015)

[25] P. Ponte, Z. Papic, F. Huveneers, and D. Abanin, Phys. Rev. Lett. 144, 140401 (2015).

[26] D. A. Abanin, W. De Roeck and F. Huveneers, (2014) arXiv:1412.4752.

[4] E. Canovi, D. Rossini, R. Fazio, G. E. Santoro, and A. Silva, Phys. Rev. B 83, 094431 (2011).

[5] M. Grifoni and P. Hanggi, Phys. Rep. 304, 229 (1998).

[6] A. Das, Phys. Rev. B 82, 172402 (2010)
[30] Energy-resolved level statistics (data not shown) does not indicate the existence of a mobile region in the spectrum for this particular model.

[31] Actually, due to the spatially exponentially localised nature of the conserved quantities, driving couples blocks at all instances by exponentially weakly; this however does not affect our argument.

[32] Note that we are only interested in perturbations much weaker than the disorder-the opposite limit might be qualitatively different (see for example [24]).

[33] A simple example of driving faster than this is given in the Supplemental Material, which includes Refs. [4]6]. 


\section{DETERMINATION OF THE TRANSITION}

To accurately locate the localisation-delocalisation transition for the undriven system, we first study the level statistics of the eigenvalues of $H_{0}$, as for example in Ref. [1]. That is, after obtaining the (quasi-)energies $\epsilon_{n}$, we calculate the following ratio involving adjacent level spacings $\delta_{n}=\epsilon_{n}-\epsilon_{n+1}$ : $r_{n}=\min \left(\delta_{n}, \delta_{n-1}\right) / \max \left(\delta_{n}, \delta_{n-1}\right)$. The mean $\eta=$ $\int_{0}^{1} d r r P(r)$ distinguishes between Wigner-Dyson-type and Poisson statistics. We calculate $\eta$ for a sequence of system sizes and extrapolate the limit of $\eta$ as $L \rightarrow \infty$.

To obtain the frequency $\omega$ above which delocalisation sets in for a driven system, we again calculate the mean of the distribution function of the quasienergy statistics $\eta=\int_{0}^{1} d r P(r)$ as a function of disorder amplitude $w$, averaged over 10000 disorder realisations and for several system sizes. Typical results are shown in Fig. 5. The transition is located at the crossing point of the lines for different system sizes: if increasing system size results in larger $\eta$ then we conclude that the system is delocalised, since $\eta=\eta_{C U E}$ for a delocalised system and $\eta=\eta_{P}$ for a localised system with $\eta_{P}<\eta_{C U E}$. Here $\eta_{C U E}$ is the value for the CUE ensemble [3].

To ensure that our results are applicable to the thermodynamic limit we need to take a frequency low enough so that the width of the energy spectrum of the undriven Hamiltonian is larger than the driving frequency $\omega$. The main practical problem is the following: with decreasing disorder amplitude $w$ and for fixed system size, the value $\omega_{c}$ increases while the energetic width of the DOS decreases (see Sec. IV). Since $\omega$ must be small compared to the width in order for our extrapolation to the thermodynamic limit to be meaningful, the $\omega_{c}$ for values of the disorder close to $w_{c}$ are inaccessible for the system sizes available to us. The width of the DOS is indicated in Fig. 5 by vertical lines; the crossing point of the curves cannot lie to the right of this line, since otherwise the finite size of the system would be important (and thus the results would not be reliable in the thermodynamic limit).

Fig. 5 reveals the following features: for $w / J \leq 6$ (where the undriven system is delocalised) the lines for succesive, increasing $L$ do not cross for values of $\omega$ below the bandwidth, indicating that the thermodynamic limit is delocalised, as expected. For $w / J>6$, there is a clear crossing point, which indicates the position of the transition. The crossing value of $\omega$ determined by this method is plotted as a function of $w / J$ in Fig. 1 in the main text.

\section{EIGENSTATE EXPECTATION VALUES}

As discussed in Ref. [2] and the main text (Fig. 2 of the main text), periodically-driven ergodic (or delocalised) systems develop a peak in the probability distribution of the eigenstate expectation values (EEVs). Fig. 6 shows explicit examples of the EEVs in the case of slow driving (system remains localised, left panel) and fast driving (system delocalises, right panel).

\section{SINGLE-PARTICLE LOCALISATION LENGTH}

In Fig. 7 we show the single-particle localisation length as a function of the eigenstate energy for the noninteracting Hamiltonian

$$
H_{0}=-\frac{1}{2} J \sum_{i=1}^{L-1}\left(b_{i}^{\dagger} b_{i+1}+b_{i+1}^{\dagger} b_{i}+h c\right)+\sum_{i=1}^{L} U_{i} n_{i}
$$

with $U_{i}$ an on-site random potential uniformly distributed between $-w$ and $+w$ and we take $w / J=5$. This plot demonstrates that, for the values of $w / J$ where the interacting Hamiltonian of the main text is in the MBL phase, the single-particle localisation length is well below the accessible system sizes. This ensures that the finiteness of this length is not a source of finite-size effects.

\section{WIDTH OF SPECTRUM OF LOCAL MODEL}

The density of states (DOS) for a large class of systems with bounded local Hilbert spaces, including the type we study, is Gaussian: $d(\epsilon)=$ $\mathcal{N}^{-1} \exp \left(-\left(\epsilon-\epsilon_{0}\right)^{2} / 2 \epsilon_{w}^{2}\right)$ [4]. An example for a particular disorder realisation of our HCB model is shown in Fig. 8, while a plot of the fitted width $\epsilon_{w}$ as a function of disorder amplitude $w$ is shown in Fig. 9. Ref. 4] shows that, in the absence of disorder, $\epsilon_{w} / J \propto L$, while Fig. 9 suggests that in the presence of strong disorder $\epsilon_{w} / J \propto L^{1 / 2}$. This may be understood via the central limit theorem: for strong disorder, the system's eigenvalues are approximately sums of uniformly distributed random numbers (the random potential at each site), and the probability distribution of a sum of $L$ uniformly distributed random numbers approaches a normal distribution with width $L^{1 / 2}$. In any case, in Fig. 5 we use the actual values of $\epsilon_{w} / J$ obtained by fitting and averaging over a number of realisations.

\section{DRIVEN TWO-LEVEL SYSTEM}

To illustrate how the energy bandwidth of a system sets a natural frequency above which driving has little effect we consider a model with finite bandwidth, namely 


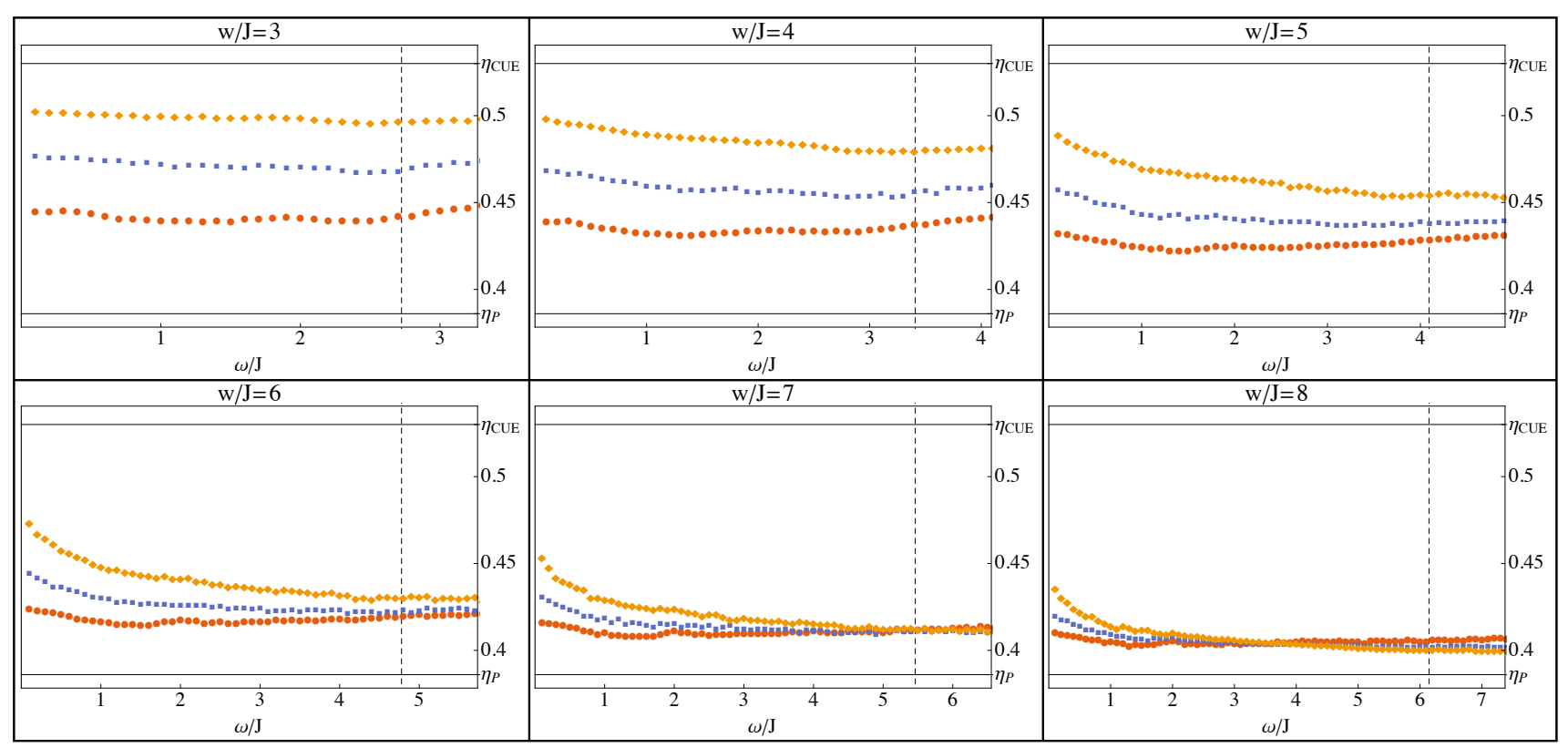

Figure 5: Level statistics for various disorder amplitudes $w / J$ as a function of driving frequency $\omega$. The driving amplitude is $\delta / J=0.1 \ll w / J, \omega / J$, and each point represents an average over 10000 disorder realisations. The dashed vertical lines indicate half the width of the energy spectrum; for $\omega$ greater than this, our results cannot be extrapolated to the thermodynamic limit (see IV). The colours correspond to system sizes $L=8,10,12$ from bottom to top for the smallest $\omega$. The values $\eta_{G O E}$ and $\eta_{P}$ correspond to the presence and absence of level repulsion, respectively, which in turn correspond to localised and delocalised phases. The dotted vertical lines correspond to the typical spectral width of the system, for frequencies above which our results cannot be used to infer the thermodynamic limit.

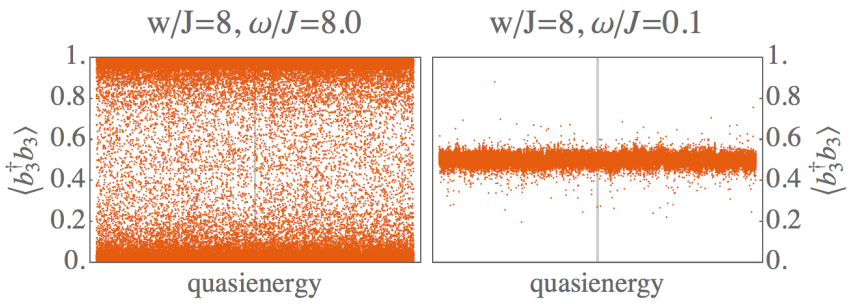

Figure 6: Plots of eigenstate expectation values (EEV) of the density at a single arbitrarily chosen site in all the eigenstates of $H_{\text {eff }}$ for a system with $w / J=8.0$, size $L=18$ for a Hilbert space dimension of $D_{H}=48620$ and driving amplitude $\delta / J=0.1$. For driving frequency above the blue line in Fig. 1 of the main text, $\omega / J=8.0$ (left), the EEVs fluctuate wildly between different eigenstates of $H_{e f f}$. In contrast, for a driving frequency below the blue line, $\omega / J=0.1$ (right), there is markedly less eigenstate-to-eigenstate variation, consistent with all states being fully mixed. This is the expected behaviour of the EEVs for clean (therefore delocalised) driven systems (see Ref. [2]). In the undriven system the EEVs appear qualitatively similar to those in the left panel.

the two-level system (TLS) [5], driven with a small amplitude:

$$
H_{T L S}=\left(\begin{array}{cc}
h & \delta \cos (\omega t) \\
\delta \cos (\omega t) & -h
\end{array}\right)
$$

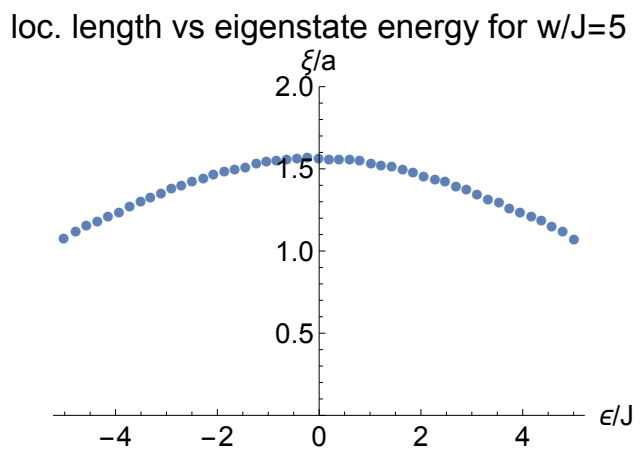

Figure 7: Single-particle localisation length for a noninteracting Anderson problem with parameters similar to the typical values used in our main text. The localisation length is well below most of the system sizes we have used.

This has a threshold frequency above which it does not react to the driving. The simplest way to see this is to directly calculate

$$
\exp \left(-i H_{\text {eff }}(\epsilon) T\right)=\mathcal{T} \exp \left(-i \int_{\epsilon}^{\epsilon+T} H_{T L S}(\tau) d \tau\right)
$$

which describes the time evolution over one period. If $H_{e f f} \approx\left(\begin{array}{cc}h & 0 \\ 0 & -h\end{array}\right)=T^{-1} \int_{0}^{T} d t H_{T L S}(t)$ (compare to Eq.7) then clearly driving has very little effect on the sys- 


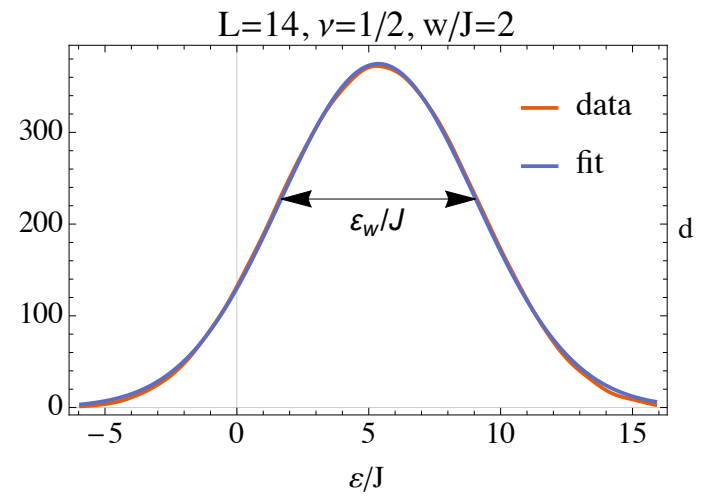

Figure 8: Comparison between actual density of states and Gaussian fit.

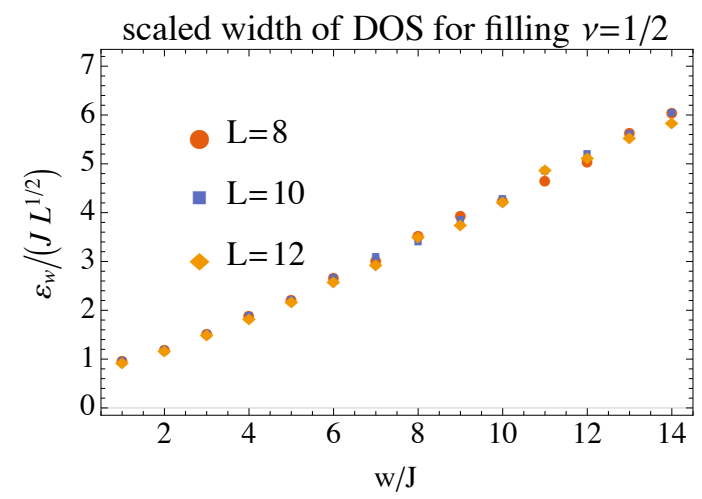

Figure 9: Width of DOS as a function of disorder amplitude for two system sizes $L=10,12$. This was obtained by explicitly calculating the DOS for 100,400 realisations of the disorder for $L=12,10$, respectively.

tem. Fig. 10 shows the Frobenius norm $\left|H_{\text {eff }}-H_{T L S}^{0}\right|$ where $H_{T L S}^{0}$ is the undriven TLS Hamiltonian (Eq. 7 with $\delta=0)$. This figure shows that the norm vanishes as a power law of $\omega / h$.

The situation for (for example) a single particle hopping on a lattice (whether to nearest-neighbour sites or with some exponentially small amplitude to hop to any distance) is completely identical.

The result described here is valid for weak driving amplitude; different physics might emerge in other limits [6].

\section{DELOCALISATION VIA DEPHASING}

We show that an initial state which is an eigenstate of the Hamiltonian $H_{1}$ and driven by alternating between $H_{1}$ and $H_{2}$ (as in the protocol described in the main text for the local disordered model) spreads out to fill the entire Hilbert space if it dephases between each change of Hamiltonian. Specifically, we construct the density matrix $\rho(0)$, diagonalise it in the eigenbasis of $H_{1}$ and then discard off-diagonal elements. It is

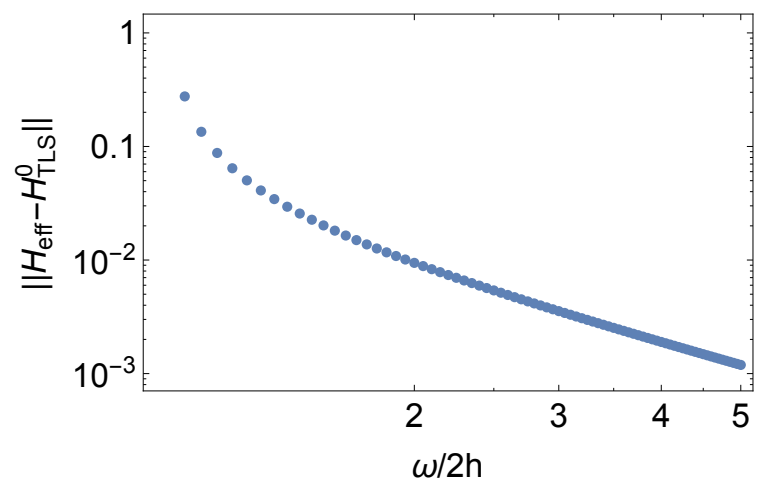

Figure 10: Approach of $H_{\text {eff }}$ to undriven $H_{T L S}$ with increasing frequency. This is the limiting form for large $h / \delta$ (weak driving); in practice, even $h / \delta=1$ gives a result indistinguishable from this.

then rotated to the eigenbasis of $H_{2}$ and, again, the off-diagonal terms are discarded. This procedure constitutes one period. Discarding the off-diagonal states is supposed to model dephasing, and is similar in spirit to the Boltzmann Stosszahlansatz. Figure ?? shows a plot of $\ln \left(\left|c_{\alpha}\right|^{2}\right)$ (the logarithimic scale is necesary as $\left|c_{\alpha}\right|^{2}$ ranges from 1 to $1 / D_{H}^{2}$ during the process, with $D_{H}$ the dimensions of Hilbert space), with $c_{\alpha}$ the projection of the state onto the eigenstates of $H_{1}$, as a function of period for an initial state that as an eigenstate. The time evolution is carried out using the driving protocol described in the main text for the local model (Eqs. 3 and 4 in the article), with the additional operation of dephasing carried out by hand (that is, off-diagonal elements in the energy basis are discarded by hand). The paramerers used are indicated in the caption.

The conclusion to be drawn from Fig. ?? is that dephasing clearly leads to the system spreading out in energy space. While not unexpected in general (a series of sudden perturbations of the system will, in general, increase its energy), this calculation confirms that the conclusion remains valid in the case of MBL Hamiltonians.

\section{THE QUANTUM RANDOM ENERGY MODEL}

Consider the set of $2^{N}$ Fock states for $N$ spin- $1 / 2$ spins defined by fixing all the $\left.\sigma_{[}^{z} i\right]$ for $i=1 \ldots N$, labelling them by $|n\rangle$ with $n=1 \ldots 2^{N}$. These form a complete basis and may be thought of as the $\left(2^{N}\right)$ vertices of an $\mathrm{N}$ dimensional hypercube. To each edge/Fock state assign an energy at random, drawn from a distribution

$$
P(E)=\frac{1}{\sqrt{\pi N}} \exp \left(-E^{2} / N\right)
$$

(ensuring extensivity of the energies, bandwidth etc). 


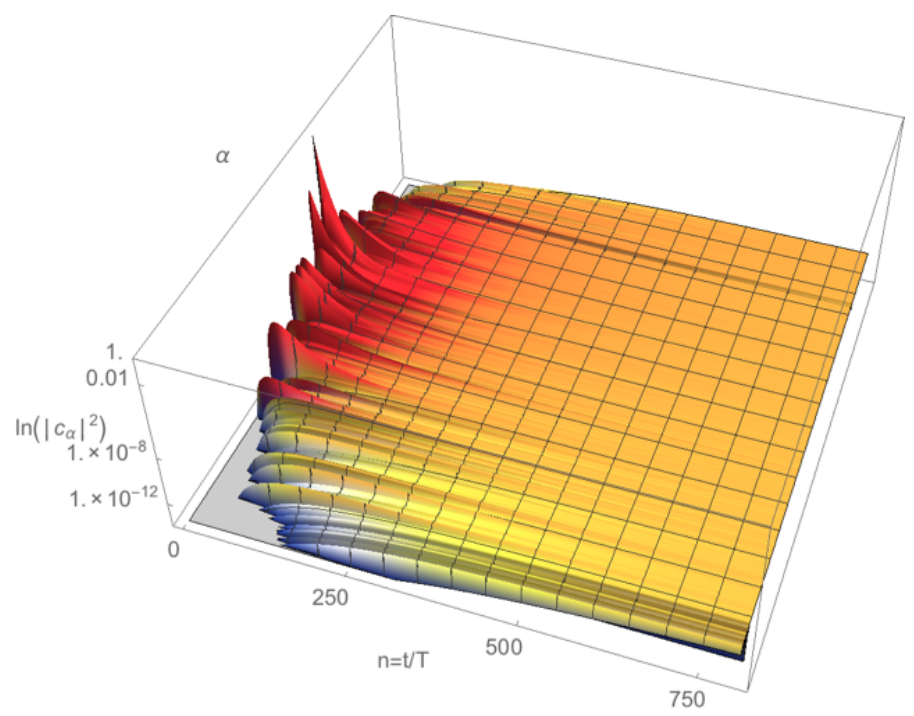

Figure 11: Stroboscopic demonstration of the spreading of an initial energy-localised state driven via alternating between two Hamiltonians and forcing dephasing by hand (see Eqs. 3 and 4 in the main text for the Hamiltonians). The parameters used are system size $L=10$ at half-filling, driving amplitude $\delta / J=0.2$, disorder amplitude $w / J=8$ (inside the localised phase) and interactions $V_{1} / J=V_{2} / J=1$. The system delocalises in energy even though both Hamiltonians are localised as a direct result of dephasing.

So far the problem is diagonal in the basis of the Fock states. Now add a term $\left.-\Gamma \sum_{i=1}^{N} \sigma_{[}^{x} i\right]$; the operator $\left.\left(N-\sum_{i=1}^{N} \sigma_{[}^{x} i\right]\right)$ is the Laplacian on the hypercube so that $\Gamma$ plays the role of a hopping amplitude. Overall, the Hamiltonian is the Anderson problem with hopping $\Gamma$ and on an $N$-dimensional hypercube. Formally, the Hamiltonian is given by

$$
\hat{H}=-\Gamma \hat{\nabla}^{2}+\hat{V}
$$

with $\hat{V}$ the random potential. This model is not local in real space, but it is local on the hypercube, ie, the state space of a spin- $1 / 2$ model.

[1] V. Oganesyan and D. Huse, Phys. Rev. B 75, 155111 (2007)

[2] A. Lazarides, A. Das, and R. Moessner, Phys. Rev. E 90, $012110(2014)$.

3] L. D'Alessio and M. Rigol, (2014), arXiv:1402.5141.

[4] E. Canovi, D. Rossini, R. Fazio, G. E. Santoro, and A. Silva, Phys. Rev. B 83, 094431 (2011).

[5] M. Grifoni and P. Hanggi, Phys. Rep. 304, 229 (1998).

[6] A. Das, Phys. Rev. B 82, 172402 (2010) 